\title{
The sandwich plug technique: Simple, effective, and rapid closure of a mechanical aortic valve prosthesis at left ventricular assist device implantation
}

\author{
William E. Cohn, MD, and O. H. Frazier, MD, Houston, Tex
}

The presence of an aortic valvular prosthesis is generally considered to be a relative contraindication to left ventricular assist device (LVAD) implantation. A bypassed, intermittently opening prosthetic valve, whether it is mechanical or biologic, carries a prohibitive risk of thromboembolic complications, despite the use of anticoagulants. ${ }^{1}$ To avoid the challenges of long-term mechanical circulatory support in patients with aortic valve prostheses, our group ${ }^{2}$ and others ${ }^{3}$ have suggested complete surgical closure of the left ventricular outflow tract (LVOT). Although suturing the leaflets together along the lines of coaptation can readily accomplish LVOT closure in patients with biologic aortic prostheses, closing the LVOT in patients with mechanical aortic prostheses generally requires a supravalvular patch. ${ }^{3}$ In this brief report, we describe a technique that enables rapid and secure closure of the LVOT in patients with mechanical bileaflet aortic valves.

In a recent series of 5 consecutive patients with aortic valve prostheses or aortic regurgitation, our team closed the LVOT at LVAD implantation, allowing the successful long-term implementation of mechanical circulatory assistance. ${ }^{2}$ Primary closure of the lines of leaflet coaptation was possible in 2 patients, 1 with a bovine prosthesis and another with a congenitally bicuspid regurgitant valve. In 2 other patients with significant native valve regurgitation, the leaflet tissue was too delicate to close primarily, so we sutured a pericardial patch around the circumference of the annulus. The fifth patient had a recently placed mechanical aortic valve. In that case, we removed the prosthesis and replaced it with a pericardial patch, as described by others. ${ }^{3}$ Although successful, this last case was technically challenging and time-consuming, leading us to develop an alternative approach to LVOT closure in patients with mechanical bileaflet aortic valve prostheses.

\section{MATERIALS AND METHODS}

For our alternative approach, we use a novel prefashioned occluder, which we construct in the operating room before starting the procedure

From the Center for Cardiac Support, Texas Heart Institute at St. Luke's Episcopal Hospital, Houston, Tex.

Disclosures: Dr Fraizer is on the Medical Advisory Board for HeartWare, Inc, Framingham, Mass.

Received for publication July 19, 2010; revisions received Oct 15, 2010; accepted for publication Nov 2, 2010; available ahead of print Jan 31, 2011

Address for reprints: William E. Cohn, MD, PO Box 20345, MC2-114A, Houston, TX 77225 (E-mail: wcohn@ @eart.thi.tmc.edu).

J Thorac Cardiovasc Surg 2011;142:455-7

$0022-5223 / \$ 36.00$

Copyright (c) 2011 by The American Association for Thoracic Surgery

doi:10.1016/j.jtcvs.2010.11.003

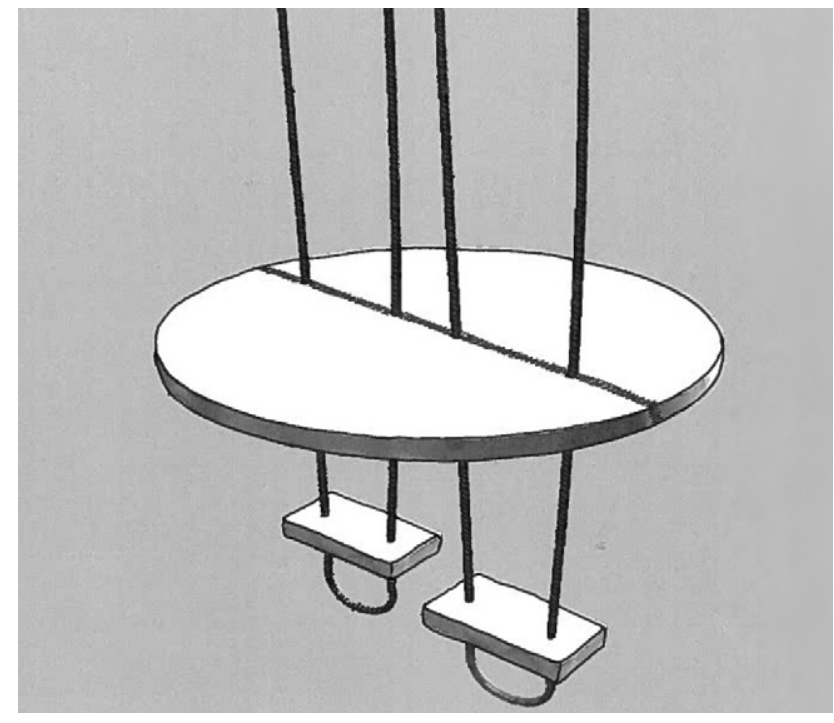

FIGURE 1. Felt circular patch is fashioned with diameter slightly larger than that of implanted mechanical valve, and line is drawn along diameter, after which 2 pledgeted Ti-Cron sutures are placed through patch such that all 4 sutures pass through line.

from polytetrafluoroethylene felt, 2.0 braided polyester sutures (Ti-Cron; Covidien, Mansfield, Mass), pledgets, and 2 large titanium vascular clips (Weck Hemoclip; Teleflex Medical, Research Triangle Park, NC). First, we fashion a circular patch of polytetrafluoroethylene felt with a diameter slightly larger than that of the implanted mechanical valve (Figure 1). The size of the mechanical aortic valve that has been implanted can generally be found in the operative report. Alternatively, a reasonably accurate measurement can be obtained by echocardiography. A prosthetic valve sizing tool can be used as a template to facilitate accurate fashioning of the felt. A diametric line is drawn on the circular patch. This will align with the edges of the closed mechanical valve leaflets once the occluder is secured in place. Two pledgeted 2.0 Ti-Cron sutures are placed through the circular patch such that all 4 sutures pass through the previously drawn diameter. The sutures should be spaced fairly widely, as illustrated in Figure 1, but care must be taken to avoid placing the sutures too close to the edge of the patch. This circular patch, which will become the innermost patch in the occluder, will be implanted below the mechanical valve prosthesis in the LVOT.

A second circular patch, the outer patch, is then fashioned to be slightly larger than the first. This will be implanted above the mechanical valve (Figure 2). A third rectangular piece of felt is then fashioned to be the width of a large titanium vascular clip and of a length that will allow the second circular patch to circumscribe it. The 2 titanium clips are secured to the rectangular piece of felt perpendicular to its long axis by applying them to the felt as one would apply them to a vascular structure. Then the rectangular felt is sutured to the second circular patch by placing a 4.0 polypropylene stitch at each of the 4 corners.

Next, a line is drawn down the center of the rectangular piece of felt along its long axis (perpendicular to the 2 titanium vascular clips), as shown 


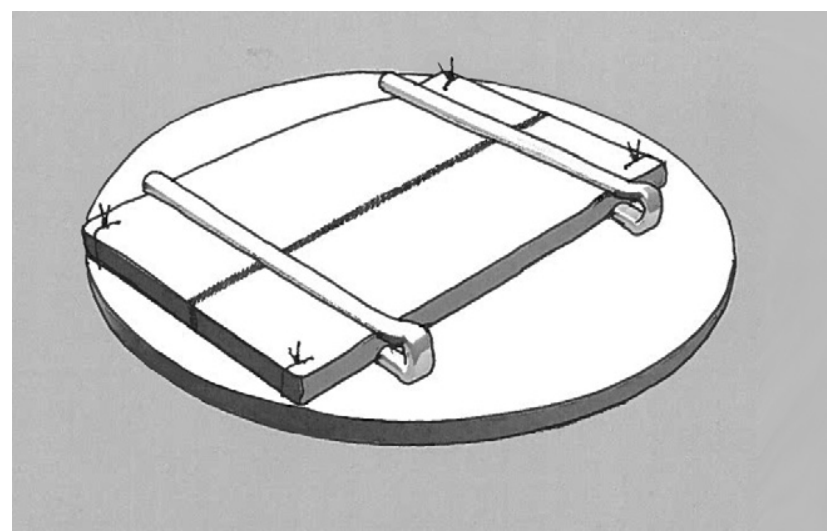

FIGURE 2. Second circular felt patch is fashioned slightly larger than first patch, and third rectangular piece of felt is attached to it with 4 interrupted sutures. Two large vascular Weck Hemoclips are secured to rectangular piece of felt as shown.

in Figure 3. All 4 ends of the 2 previously placed Ti-Cron sutures are then passed through the second patch such that they exit through the previously drawn line. Care must be taken to ensure that the sutures do not cross or become entangled between the 2 circular patches.

Implantation of the occluder requires only limited exposure of the mechanical prosthetic valve. During a brief period of aortic crossclamping, the mechanical valve leaflets are pivoted to open the valve wide (Figure 4). The lower circular patch and the 2 pledgets are then passed into the LVOT through the central orifice. A fine forceps is helpful in this maneuver. During this step, care must be taken to avoid cracking or dislodging either of the valve leaflets. Once the patch and pledgets are completely past the valve, the sutures are held in a tension-free fashion to allow the patch to drop into the left ventricle. Then light tension is applied by pulling the patch up against the underside of the bileaflet valve and pivoting the leaflets closed. The inner patch should rotate into alignment with the line of leaflet closure as a result of the line created by the 4 sutures. If the patch does not align, the previous steps should be repeated, alternating between slack and light tension until correct alignment is achieved.

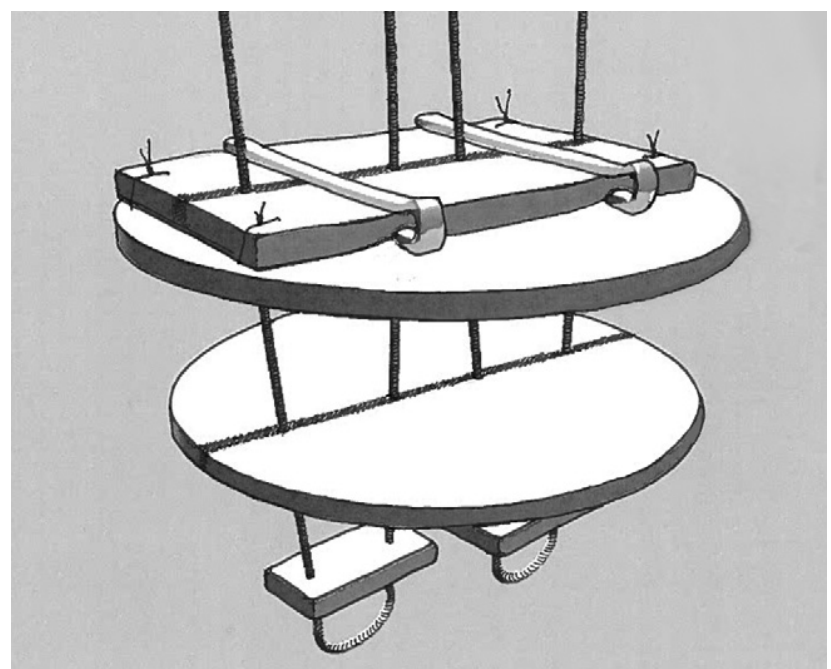

FIGURE 3. Second line is drawn down long axis of rectangular piece of felt. All 4 ends of 2 previously placed pledgeted Ti-Cron sutures are passed through second patch such that they exit through line.

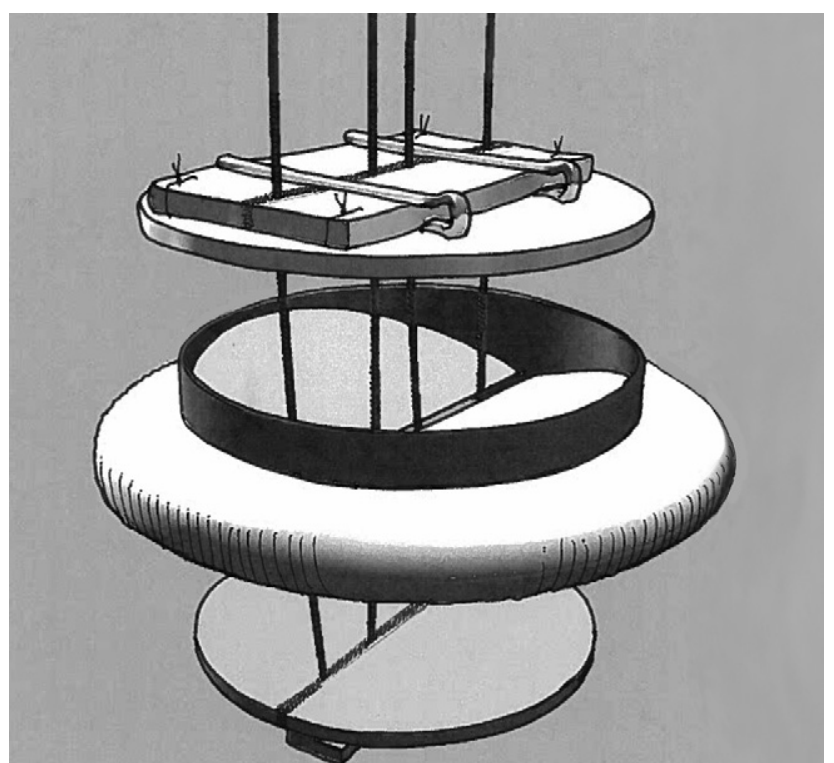

FIGURE 4. After mechanical valve leaflets are pivoted open, first circular patch and 2 pledgets are passed through central orifice. Sutures are manipulated to allow patch to drop into left ventricle, then lightly tensed to pull patch up against underside of valve and pivot leaflets closed.

Next, the second circular patch is "parachuted" into position, and the 2 sutures are tied securely (Figure 5). Because of the linear orientation of the sutures, the outer patch should rotate into position as well, such that each of the lines drawn on the felt rotates into alignment with the line of leaflet closure. In this position, the 2 large titanium vascular clips will lie perpendicular to the line of leaflet closure, and the Ti-Cron sutures, when tied, will pull downward at each clip midpoint. Because the position of each clip is fixed relative to the outer circular patch, this relationship is maintained. When the sutures are tied securely, the 2 valve leaflets are rotated into a closed position and pinned shut between the pledgets and the inner patch below the valve and the ends of the titanium clips and the outer patch above the valve (Figure 6).

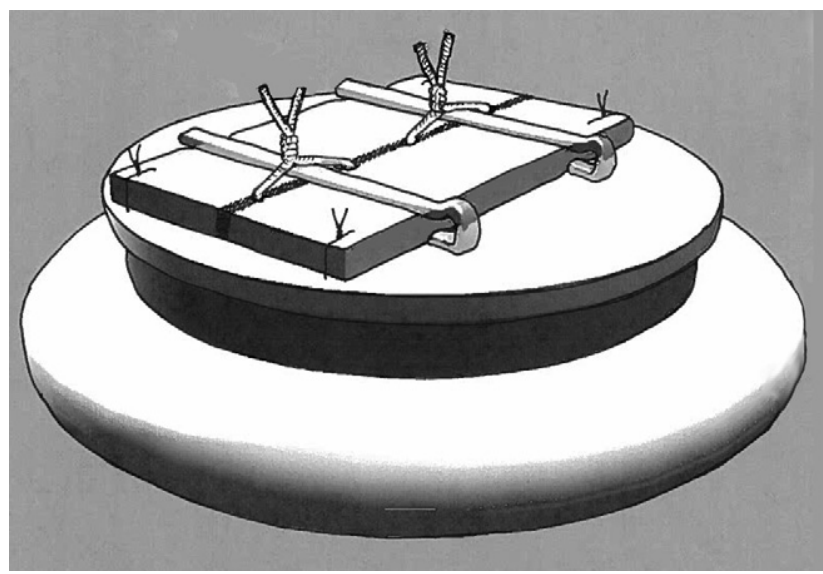

FIGURE 5. Second circular patch is "parachuted" into position, and 2 sutures are tied securely. Line drawn on rectangular piece will align with line of mechanical leaflet closure. Valve is rapidly and effectively closed such that leaflets cannot pivot, and entire prosthesis is covered with felt. 


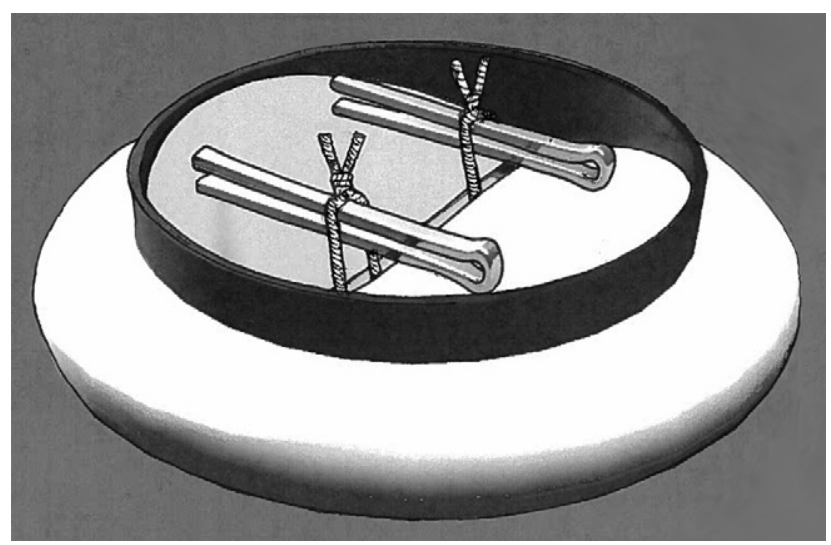

FIGURE 6. In this figure, outer patch has been removed to show geometric relationship between large Weck Hemoclips and mechanical valve leaflets. Suture tension results in upward traction on straight inner leaflet edges by pledgets and downward pressure on curved outer edges by Hemoclips, resulting in pinning of valve leaflets in closed position.

\section{RESULTS}

This technique has been used in 3 patients, each of whom had a mechanical bileaflet aortic valve prosthesis. In each case, placement of the device took only 2 or 3 minutes and echocardiography demonstrated complete closure of the LVOT without evidence of regurgitation or leaflet motion. The first patient was discharged home; he was given warfarin (Coumadin, target international normalized ratio of 2-3) for anticoagulation. He did well for 11 months, at which time he underwent elective cardiac transplant. Serial echocardiography after LVAD implantation, including an echocardiogram obtained 1 week before transplant, showed that the device was in place and that there was no regurgitation or thrombus. Photographs of the explanted heart show the device well-seated on both sides of the prosthesis without evidence of thrombus (Figure 7). The patient continues to do well now 3 months after his transplant operation. The second patient had undergone multiple previous cardiac operations. He died of sepsis and multisystem organ failure 4 weeks after LVAD implantation. An echocardiogram on postoperative day 28 showed the device in good position with no aortic insufficiency or thrombus. There was no clinical evidence of systemic emboli. This patient was maintained with intravenous heparin throughout most of his hospitalization. A third gravely ill patient in whom we implanted the felt sandwich plug died of multisystem organ failure 2 weeks after LVAD implantation. An echocardiogram on postoperative day 10 showed no insufficiency or thrombus. No thrombus was found on the felt or in the aortic root at autopsy. The mechanical valve remained fixed in the closed position.
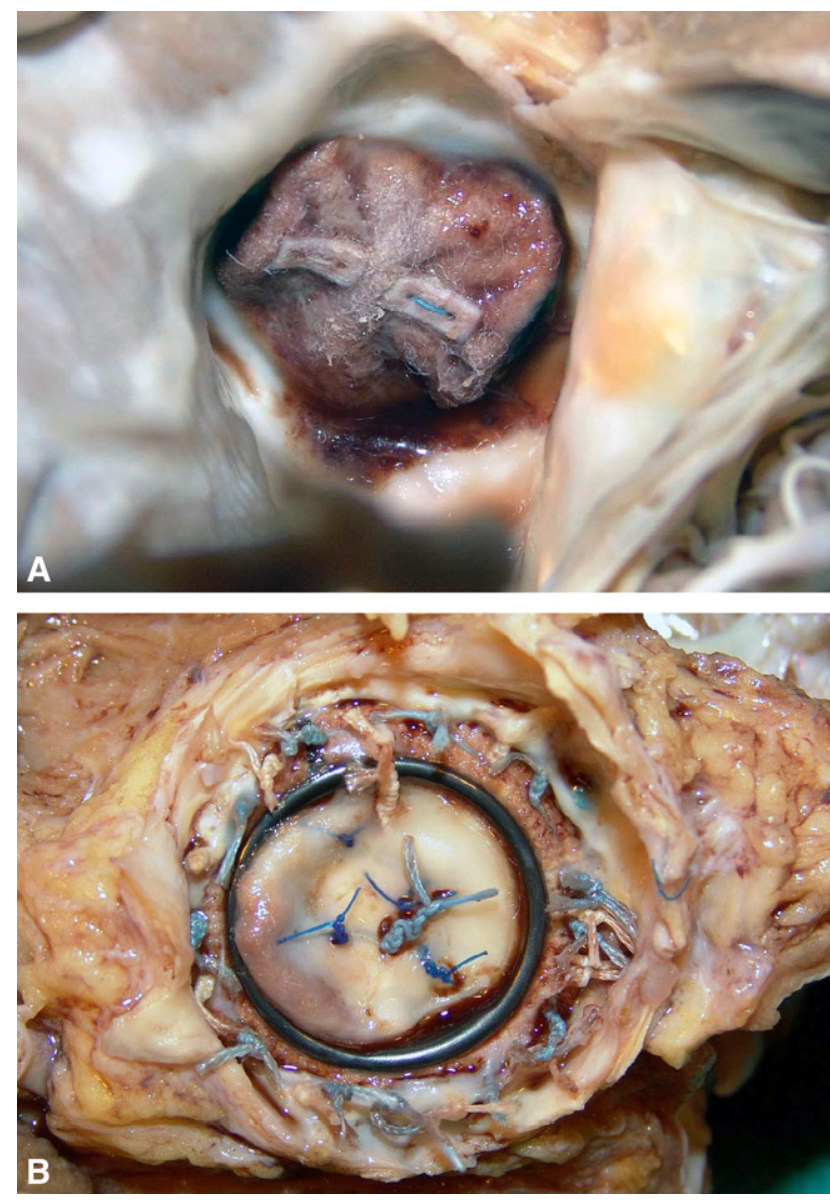

FIGURE 7. Photographs of aortic (A) and ventricular (B) aspects of left ventricular outflow tract taken at cardiac transplantation show felt sandwich in good position without evidence of thrombus.

\section{DISCUSSION}

Although the experience is limited, the ease and speed of implementation and the excellent result obtained in the lone long-term survivor are promising. We therefore now advocate this technique of LVOT closure for patients with previously implanted bileaflet aortic mechanical prostheses who undergo implantation of a long-term LVAD.

We acknowledge Marianne Mallia, ELS, of the department of Scientific Publications at the Texas Heart Institute, for editorial assistance in the preparation of the manuscript.

\section{References}

1. Myers JL, Bull A, Kastl DG, Pierce WS. Fusion of prosthetic valve during left heart bypass. J Thorac Cardiovasc Surg. 1981;82:263-7.

2. Cohn WE, Demirozu ZT, Frazier OH. Surgical closure of left ventricular outflow tract after left ventricular assist device implantation in patients with aortic valve pathology. J Heart Lung Transplant. 2011;30:59-63.

3. Pelletier MP, Chang CP, Vagelos R, Robbins RC. Alternative approach for use of a left ventricular assist device with a thrombosed prosthetic valve. J Heart Lung Transplant. 2002;21:402-4. 